\title{
MODELO GEOQUÍMICO APLICADO À AVALIAÇÃO DA QUALIDADE DE ÁGUA DA BACIA DO ALTO CURSO DO RIO PARACATU - MG
}

\author{
MULHOLLAND, D. S. ${ }^{1, *} ;$ BOAVENTURA, G. R. ${ }^{1,2}$ \& ARAÚJO, D. F. ${ }^{1,3}$ \\ 1 - Instituto de Geociências, Universidade de Brasília, Campus Universitário Darcy Ribeiro. Labora- \\ tório de Geoquímica. ICC Centro, Subsolo - CSS 401/51. Asa Norte, Brasília - DF. CEP: 70910-900; \\ 2 - grbunb@unb.br; 3 - quimico.unb@gmail.com \\ *Corresponding author: danambiental@gmail.com
}

\begin{abstract}
Mulholland, D. S.; Boaventura, G. R. \& Araújo , D. F. 2010. Geochemical model applied to water quality assessment of the upper course of the Paracatu River watershed - MG. Braz. J. Aquat. Sci. Technol. 14(2): 39-46. ISSN 1808-7035. This work reports a geochemical study of three watersheds located in the upper course of the Paracatu River (one of the most important sub-basins of the upper-middle São Francisco River). It aimed to establish a geochemical model that can assist in identifying the sources and distribution of physico-chemical components and dissolved metals in water, assessing how the local geology and human activities influence the quality of the aquatic systems. The predominant geology of the region includes the Vazante Group, basically formed by a sequence of clay and dolomitic clay where zinc and lead mineralization occurs, and the Canastra Group (Paracatu Formation) composed of a sequence of phyllites and carbonaceous phyllites with intercalations of quartzites, where gold occurs in iron and arsenic sulfides. The samples were analysed using ICP/OES, AAS, spectrophotometric (UV/Visible) techniques and volumetric methods. The development of the geochemical model used multivariate statistical analysis including principal components, multiple regression and hierarchical clustering. The proposed model determined that $\mathrm{Fe}, \mathrm{Mn} \mathrm{SO}_{4}{ }_{4}^{2-}$, and color come primarily from sulfide oxidation; $\mathrm{HCO}_{3} ; \mathrm{Ca}, \mathrm{Mg}, \mathrm{Zn}$, and $\mathrm{Ba}$, from dolomitic limestone dissolution, and $\mathrm{PO}_{4}{ }^{3-}, \mathrm{NO}_{3}{ }^{-}, \mathrm{NH}_{3}$ and turbidity from the input of urban sewage. Indices were created for each group of parameters representing the intensity of these processes and classifying the sampling sites according to their predominant chemical composition (water with low mineralization, high carbonate, or sulphate mineralization and pollution by urban sewage). The proposed model can be used in regional water quality monitoring, and as a reference for other locations.
\end{abstract}

Keywords: water quality index, environmental impact, water assessment, water pollution, multivariate analyses.

\section{INTRODUÇÃO}

Os materiais dissolvidos e particulados da água, transportados pelos sistemas de drenagem, têm composição determinada pela geologia local, cobertura vegetal e uso do solo, carregando assim, a assinatura geoquímica da região (Bricker \& Jones, 1995). A agricultura, pecuária, urbanização, mineração e remoção da cobertura vegetal causam alterações nos corpos hídricos, mudando os ciclos elementares e a dinâmica de erosão, transporte e deposição do solo (Van Andel et al., 1997). Essas alterações devem-se à fontes difusas como o escoamento superficial de áreas agropastoris, urbanas e áreas degradadas e de fontes pontuais individualizadas como despejo de esgotos urbanos e efluentes industriais (Carpenter et al., 1998).

A bacia do Rio Paracatu teve seu processo de ocupação iniciado por volta de 1730, com o surgimento de atividades relacionadas à mineração de ouro e à pecuária, sendo intensificada após a criação de Brasília. Nas bacias do córrego Rico, ribeirão Santa Catarina e rio Escuro, afluentes do alto rio Paracatu, encontramse, atualmente, pequenas cidades, extensas áreas de pecuária, agricultura de sequeiro e irrigada, regiões com plantio de eucalipto e pinheiro e áreas degradadas pela mineração (Brasil, 1998). Os municípios de Paracatu,
Guarda-Mor, Vazante e Lagamar, encontrados dentro da área de estudo, apresentam, respectivamente, $75.216,6.656,18.928$ e 7.710 habitantes, com população predominantemente urbana. Não possuem rede de coleta e tratamento de esgoto e lixo que atenda todos os habitantes (CNM, 2007). Devido à presença de depósitos minerais de ouro, willemita $\left(\mathrm{Zn}_{2} \mathrm{SiO}_{4}\right)$, calamina $\left(\mathrm{Zn}_{4} \mathrm{Si}_{3} \mathrm{O}(\mathrm{OH})_{2}\right)$, galena (PbS) e esfalerita $(\mathrm{ZnS})$, mineradoras de ouro (Morro do Ouro, Paracatu), chumbo e zinco (Morro Agudo e Vazante) também acorreram à região (Brasil, 1988).

Os prognósticos do plano diretor dos recursos hídricos da bacia hidrográfica do rio Paracatu (Brasil, 1996) apontam para um cenário efetivo crítico, principalmente, em relação aos efluentes das atividades de mineração que são intensas na região. A ausência de redes de coleta e tratamento de esgoto e lixo que atendam toda população também é apontada como uma ameaça à qualidade da água.

Para avaliar a correta influência das atividades antrópicas nos sistemas aquáticos Ott (1978) e Ball \& Church (1980) sugerem a utilização de índices e modelos de qualidade de água. Para Haase et al. (1989), os modelos que melhor representam as variações e alterações no meio são baseados em métodos estatísticos, já que permitem a melhor definição dos parâmetros 
indicadores de acordo com a região de estudo. Modelos não estatísticos apresentam um grau de subjetividade elevado, pois dependem da escolha teórica das variáveis que atuarão como os principais indicadores das alterações no meio aquático (Toledo \& Nicolella, 2002).

Neste trabalho é apresentado o estudo geoquímico de três importantes bacias do rio Paracatu com o objetivo de criar um modelo geoquímico que auxilie identificar as fontes dos compostos físico-químicos e metais dissolvidos na água, definir quais parâmetros são indicadores da qualidade da água, quais os melhores índices a serem utilizados para determinar a intensidade dos processos hidrogeoquímicos e diferenciar os tipos de água de acordo com sua composição.

\section{ÁREA DE ESTUDO}

Está localizada no alto curso da bacia do rio Paracatu (uma das mais importantes sub-bacias do altomédio São Francisco), no extremo noroeste do estado de Minas Gerais, entre as coordenadas 46 18'50" e

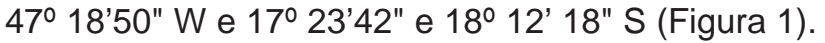
Representa as bacias hidrográficas do rio Escuro, ribeirão Santa Catarina e córrego Rico com áreas de, aproximadamente, 4850, 1726 e 900 km² $^{2}$ comprimento do rio principal de 108,86 e $57 \mathrm{~km}$, respectivamente. O clima da região é classificado como megatérmico chuvoso do tipo Aw (clima quente e úmido com chuvas de verão). O período chuvoso abrange os meses de outubro a abril e o período seco de junho a setembro. Encontra-se na zona externa meridional da Faixa de Dobramentos Brasília dentro das unidades geológicas do Grupo Vazante e Canastra.

Os metassedimentos do Grupo Vazante são formados, basicamente, por uma sequência argilosa e argilo-dolomítica com estromatólitos de barreira recifal. É interpretado como sedimentação de margem passiva neoproterozóica na borda oeste do Cráton São Francisco. As formações deste grupo, encontradas na área de estudo, são: 1) Morro do Calcário: caracterizada pela presença de dolomitos róseos estromatolíticos, dolarenitos e doloruditos que hospedam as mineralizações de chumbo e zinco (galena e esfalerita), em Morro Agudo; 2) Serra do Poço Verde: sequência de dolomitos algais com mineralizações de zinco (willemita e calamina), em Vazante; 3) Lagamar: unidade psamo-pelítica carbonática, representada por alternância de conglomerados, quartzitos, metassiltitos e ardósias; 4) Serra do Garrote: espesso pacote de ardósias com finas intercalações de quartzitos; 5) Serra da Lapa: representada por filitos carbonosos, metassiltitos carbonáticos, lentes dolomíticas e quartzitos (Valeriano et al., 2004).

O Grupo Canastra é constituído por rochas metassedimentares siliciclásticas, compostas por camadas de filitos carbonosos (Formação Paracatu), que cedem lugar a pacotes de quartzitos e filitos cloríticos

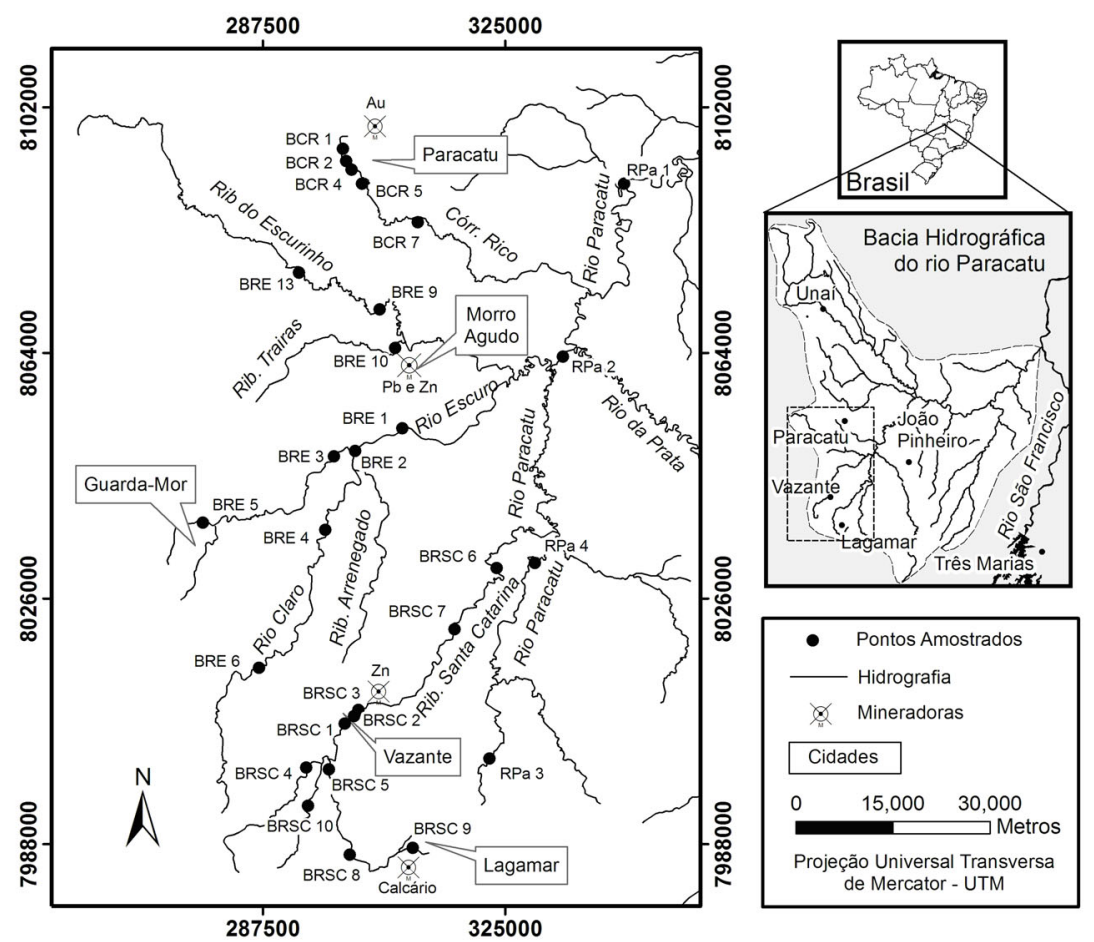

Figura 1 - Mapa de localização da área de estudo e dos pontos de coleta de água. Siglas: BRE - Bacia do rio Escuro. RPa- Rio Paracatu. BRSC - Bacia do Rio Santa Catarina. BCR - Bacia do Córrego Rico. 
e sericíticos no topo (Campos Neto, 1984 e FreitasSilva \& Dardenne, 1992). A mineralização aurífera em Morro do Ouro ocorre em sulfetos de ferro ou arsênio, apresentando ouro residual, resultante da decomposição dos sulfetos ou livre em boudins de quartzo (Brasil, 1988).

\section{MATERIAL E MÉTODOS}

\section{Coleta e análise da água}

As amostras de água superficial foram coletadas durante a estação seca (setembro de 2007), em vinte e oito pontos distribuídos nas bacias hidrográficas estudadas. A localização dos pontos foi definida de acordo com as diferentes unidades geológicas e os diversos tipos de uso do solo.

Alíquotas das amostras foram filtradas, utilizando membranas HA em ésteres de celulose, Millipore, estéril, com $0.45 \mu \mathrm{m}$ de poro e, posteriormente, acidificadas com $\mathrm{HNO}_{3}(10 \% \mathrm{v} / \mathrm{v})$ para determinação de metais. Os elementos $\mathrm{Ca}, \mathrm{Mg}, \mathrm{Mn}, \mathrm{Fe}, \mathrm{Ba}$ e $\mathrm{Zn}$ foram determinados usando espectrometria de emissão óptica com plasma indutivamente acoplado (ICP/OES) da marca Spectro Analytical Instrumental $\mathrm{GmbH}$, modelo Spectroflame FVM03, equipado com monocromador com rede holográfica de 2400 estrias $/ \mathrm{mm}$ e policromadores no vácuo e no ar. Os elementos $\mathrm{Na} \mathrm{e} \mathrm{K}$ foram determinados por espectrometria de emissão atômica em espectrofotômetro, marca Perkin Elmer, modelo 603 de duplo feixe. As análises dos metais foram validadas por amostras certificadas NIST - National Institute of Standards and Tecnology (NIST 1643d e 1640). Os parâmetros físico-químicos $\mathrm{PO}_{4}{ }^{3-}, \mathrm{SO}_{4}{ }^{2-}, \mathrm{NO}_{3}$ , $\mathrm{NH}_{3}$, cor real e turbidez foram determinados por técnicas colorimétricas com leitura em espectrofotômetro (Hach modelo DR2500). Os métodos utilizados foram: $\mathrm{PO}_{4}{ }^{3-}$ (Ascorbic Acid Method), $\mathrm{SO}_{4}{ }^{2-}$ (SulfaVer4 Method), $\mathrm{NO}_{3}^{-}$, (Cadmium Reduction Method), $\mathrm{NH}_{3}$ (Nessler Method), cor real (Platinum-Cobalt Method), turbidez (Absorptometric Method), alcalinidade (Volumetric Method) e $\mathrm{Cl}^{-}$(Argentometric Method). Cabe ressaltar que para alcalinidade foram determinados $\mathrm{HCO}_{3}, \mathrm{CO}_{3}{ }^{2-}$ $\mathrm{e}_{2} \mathrm{CO}_{3}$, sendo que apenas $\mathrm{HCO}_{3}{ }^{-}$apresentou concentrações não nulas. No campo foram determinados condutividade elétrica (C.E.) e pH, utilizando um multiparâmetro (Hach - Sension 156).

\section{Tratamento estatístico}

A Análise de Principais Componentes (APC) foi realizada segundo Farnham et al. (2003), onde os pesos dos parâmetros (factor loading) e os escores das amostras (sample factor score) foram calculados de acordo com a rotação varimax (Vondouris et al., 1997) e as componentes que obtiveram autovalores (eingenvalues) maiores que um foram selecionadas. A regressão múltipla foi realizada de acordo com Davis (1986), utilizando o método foward selection. O agrupamento hierárquico utilizou o método de Ward (1963), tendo como euclidiana a medida de distância. Com o intuito de não perder informações, as concentrações que se situaram abaixo do limite de detecção (LD) do ICP/OES foram substituídas pela metade do valor do LD para o respectivo ponto amostrado (Petersen et al., 2001 e Chen et al., 2007). Os pontos que apresentaram concentrações abaixo do LD foram BRE1, BRE2, BRE3, BRE4, BRE5, BRE6, RPa4, BCR2 e BCR7 para Zn e BRE3 para Ba. A definição dos pontos que apresentaram concentrações anômalas (média $+2 \sigma$ ) foi determinada de acordo com o tipo de distribuição que melhor se ajusta a cada um dos parâmetros. Através do teste de Kolmogorov-Smirnov pode-se definir que os parâmetros $\mathrm{NH}_{3}, \mathrm{NO}_{3}$, $\mathrm{PO}_{4}^{3-}, \mathrm{SO}_{4}^{2-}$, $\mathrm{Ca}$ e $\mathrm{Mn}$ ajustam-se melhor a uma distribuição lognormal, enquanto cor, turbidez, C.E., $\mathrm{HCO}_{3}{ }^{-}$, pH, Cl, K, Na, Mg, Fe, Ba e Zn, a uma distribuição normal. A Tabela 1 apresenta a média e o desvio padrão dos resultados obtidos, assim como comparações com demais bacias hidrográficas e com padrões de qualidade de água.

\section{RESULTADOS E DISCUSSÃO}

\section{Identificação das fontes}

A APC foi aplicada nos resultados obtidos nas análises geoquímicas para avaliar a correlação conjunta dos parâmetros analisados, determinando um agrupamento entre eles, de forma a facilitar a compreensão de suas origens. Este método resumiu todos os parâmetros analisados em três principais componentes (PC), representando $81 \%$ da variância total dos dados. O PC1 obteve elevados pesos (>0.7) de $\mathrm{HCO}_{3}$, Ca, Mg, Ba, Zn e C.E., representando $40 \%$ da variância; o PC2 de $\mathrm{NH}_{3}, \mathrm{NO}_{3}$, $\mathrm{PO}_{4}{ }^{3-}$ e turbidez, representando $30 \%$ da variância e o $\mathrm{PC}^{4}$ de $\mathrm{SO}_{4}^{2-}$, $\mathrm{Fe}, \mathrm{Mn}$ e cor, representando $11 \%$ da variância (Figura 2).

O PC1 representa o processo de dissolução de rochas carbonáticas, responsável pela produção de águas bicarbonatadas cálcicas-magnesianas (tipo Ca-

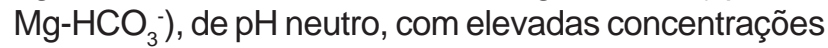
de $\mathrm{Ca}, \mathrm{Mg}, \mathrm{Ba}, \mathrm{Zn}, \mathrm{HCO}_{3}^{-}$e, consequentemente, elevada C.E.. O Ba e $Z n$ são elementos-traço típicos de minerais instáveis como a calcita e dolomita (AndrewJones, 1968) e das mineralizações de $\mathrm{Zn}$ e Pb, apresentando baixa mobilidade devido o $\mathrm{pH}$ levemente alcalino (Rose et al., 1979).

O PC2 agrupou $\mathrm{NH}_{3}, \mathrm{PO}_{4}{ }^{3-}, \mathrm{NO}_{3}^{-}$e turbidez evidenciando uma contribuição antrópica. Segundo Pieterse et al. (2003), os efluentes das áreas urbanas 
Mulholland et al.: Geoquímica na avaliação da qualidade da água.

Tabela 1 - Concentrações (média $\pm \sigma$ ) dos parâmetros analisados em cada bacia hidrográfica estudada. Todos os dados estão em mg/ L com exceção de cor (mg/L PtCo), turbidez (FTU), C.E. $(\mu \mathrm{S} / \mathrm{cm})$ e pH. Siglas: BRE - Bacia do rio Escuro; RPa- Rio Paracatu; BRSC Bacia do Ribeirão Santa Catarina; BCR - Bacia do Córrego Rico; VMP - Valores máximos permitidos para água potável (Brasil, 2004); BRSB - Bacia do Rio São Bartolomeu, D.F. (Boaventura et. al., 1998) e BRO - Bacia do rio Odiel, Espanha (Olías et. al., 2004).

\begin{tabular}{|c|c|c|c|c|c|c|c|}
\hline & \multicolumn{4}{|c|}{ Área de Estudo } & \multicolumn{3}{|c|}{ Comparações } \\
\hline & $B C R$ & $B R E$ & BRSC & $R P a$ & VMP & $B R S B$ & BRO \\
\hline NH3 & $0.93 \pm 1.29$ & 0.00 & $0.13 \pm 0.31$ & $0.03 \pm 0.02$ & 1.50 & 0.16 & 1.49 \\
\hline NO3 & $1.54 \pm 2.27$ & $0.34 \pm 0.34$ & $0.37 \pm 0.28$ & $0.15 \pm 0.13$ & 10 & 0.15 & 5.5 \\
\hline SO4 & $15.8 \pm 7.82$ & $0.78 \pm 0.67$ & $0.60 \pm 0.70$ & $1.25 \pm 0.50$ & 250 & -- & 1204 \\
\hline PO4 & $0.50 \pm 0.67$ & $0.12 \pm 0.09$ & $0.21 \pm 0.16$ & $0.14 \pm 0.07$ & -- & 0.12 & -- \\
\hline Cor & $23.0 \pm 10.7$ & $16.3 \pm 10.1$ & $9.50 \pm 8.67$ & $10.0 \pm 8.12$ & 15 & -- & -- \\
\hline Turb & $12.6 \pm 8.59$ & $9.00 \pm 2.29$ & $5.80 \pm 3.19$ & $11.8 \pm 5.56$ & 5 & -- & -- \\
\hline $\mathrm{HCO} 3$ & $26.9 \pm 18.4$ & $25.9 \pm 29.6$ & $99.9 \pm 28.2$ & $38.6 \pm 12.4$ & -- & 2.80 & -- \\
\hline C.E. & $58.1 \pm 23.3$ & $42.2 \pm 54.6$ & $168 \pm 53.05$ & $49.9 \pm 25.2$ & -- & 20.5 & 194 \\
\hline$p H$ & $6.66 \pm 0.17$ & $7.00 \pm 0.47$ & $7.58 \pm 0.30$ & $7.39 \pm 0.29$ & $6.0-9.5$ & 6.00 & 3.07 \\
\hline $\mathrm{Cl}$ & $5.81 \pm 0.97$ & $3.73 \pm 1.49$ & $3.86 \pm 0.82$ & $2.66 \pm 0.20$ & 250 & -- & 23.6 \\
\hline$K$ & $1.22 \pm 0.84$ & $0.61 \pm 0.62$ & $1.24 \pm 0.38$ & $1.17 \pm 0.27$ & -- & 1.18 & 3.20 \\
\hline $\mathrm{Na}$ & $1.09 \pm 0.82$ & $0.54 \pm 0.57$ & $1.00 \pm 0.69$ & $1.24 \pm 0.65$ & 200 & 1.65 & 26.6 \\
\hline $\mathrm{Ca}$ & $3.14 \pm 1.36$ & $3.12 \pm 4.86$ & $20.49 \pm 6.15$ & $5.79 \pm 1.90$ & -- & 4.20 & 112 \\
\hline Mg & $2.47 \pm 1.02$ & $1.97 \pm 3.36$ & $8.39 \pm 2.97$ & $2.39 \pm 1.04$ & -- & 1.52 & 94.0 \\
\hline$M n$ & $0.09 \pm 0.02$ & $0.02 \pm 0.01$ & $0.02 \pm 0.01$ & $0.03 \pm 0.03$ & 0.1 & -- & 13.7 \\
\hline $\mathrm{Fe}$ & $0.36 \pm 0.07$ & $0.18 \pm 0.06$ & $0.11 \pm 0.07$ & $0.17 \pm 0.04$ & 0.3 & 0.42 & 23.47 \\
\hline $\mathrm{Ba}$ & $0.012 \pm 0.005$ & $0.008 \pm 0.009$ & $0.042 \pm 0.017$ & $0.018 \pm 0.003$ & 0.7 & -- & -- \\
\hline$Z n$ & $0.017 \pm 0.013$ & $0.016 \pm 0.014$ & $0.029 \pm 0.010$ & $0.012 \pm 0.009$ & 5 & -- & 24.23 \\
\hline
\end{tabular}

são uma das principais fontes de entrada de nitrogênio e fósforo nos sistemas aquáticos.

O PC3 representa o processo de oxidação da pirita, onde ocorre a liberação de $\mathrm{SO}_{4}{ }^{2-}$ e de íons $\mathrm{H}^{+}$ (Litch, 1998), produzindo águas sulfatadas de $\mathrm{pH}$ ácido, aumentando a mobilidade de Fe e Mn. Entretanto, as amostras estudadas não apresentaram $\mathrm{pH}$ inferior a 5.94, sugerindo a ocorrência de uma rápida neutralização por carbonatos. $\mathrm{SO}_{4}{ }^{2-}$, Fe e Mn mostraram ser importantes fatores que causam o aumento da cor da água, podendo atingir $35 \mathrm{mg} / \mathrm{L}$ (Pt-Co).

Os elementos $\mathrm{K}$ e $\mathrm{Na}$ foram reunidos em dois grupos por apresentarem pesos maiores que 0.5 nos PC's 1 e 2 (Figura 2 a e b). No PC 1, o Na e K têm origem no processo de intemperismo, principalmente da hidrólise de minerais primários, como alguns feldspatos e micas, que são convertidos em minerais neoformados, geralmente um argilomineral, acompanhado da solubilização de K ou Na no meio aquoso. No PC 2 , estes elementos, juntamente com o $\mathrm{Cl}^{-}$, estão associados à entrada dos esgotos domésticos nos corpos hídricos. De acordo com CETESB (1974) e Martinelli et al. (2004) tais elementos podem ser introduzidos nos sistemas aquáticos na forma de sais dissolvidos, presentes em água residuais de áreas urbanas.

Os dados contidos na APC permitiram a identificação das fontes dos parâmetros analisados, resumindo a assinatura geoquímica da região em três grupos: 1) rochas carbonáticas (C.E., $\mathrm{Ca}, \mathrm{Mg}, \mathrm{HCO}_{3}$, $\mathrm{Ba}, \mathrm{Zn}$,
$\mathrm{Na}$ e K); 2) esgoto doméstico $\left(\mathrm{NH}_{3}, \mathrm{NO}_{3}^{-}, \mathrm{PO}_{4}^{3-}\right.$, turbidez, $\mathrm{Cl}^{-}, \mathrm{Na}$ e K) e 3) minerais sulfetados $\left(\mathrm{SO}_{4}{ }^{2-}, \mathrm{Fe}, \mathrm{Mn}\right.$ e cor).

\section{Índice geoquímico}

Os índices geoquímicos foram determinados a partir da adaptação do método utilizado por Shoji et al. (1966) e Lohani \& Mustapha (1982). A regressão múltipla foi utilizada para criar modelos matemáticos que expressem a intensidade de cada processo determinado na interpretação da APC, de forma a predizer os escores das amostras através de uma equação linear, demonstrando os melhores indicadores que controlam os processos anteriormente citados. Os escores das amostras (sample factor scores), obtidos na APC, foram utilizados como variáveis dependentes, sendo modelados por funções lineares compostas pelo menor número de parâmetros possíveis e que apresentem maior correlação com a variável a ser predita.

De acordo com a Tabela 2, observa-se que os índices determinados explicam $96.1 \% ; 96.1 \%$ e $89.3 \%$ da variância dos grupos (G) 1, 2 e 3, respectivamente, apresentando o $\mathrm{F}$ calculado consideravelmente maior que o $\mathrm{F}$ crítico com seus respectivos graus de liberdade (g.l.). Os pesos padronizados (Z) determinam a relevância dos parâmetros no cálculo do índice.

O I determina a intensidade da mineralização da água devido à dissolução de rochas carbonáticas, demonstrando que $\mathrm{Mg}, \mathrm{Zn}$ e Ba são os melhores ele- 
Tabela 2 - Índices calculados para cada grupo geoquímico. Equações I utilizam dados com concentrações em mg/L com exceção de cor (mg/L PtCo) e turbidez (FTU) e equações Z utilizam dados padronizados.

\begin{tabular}{|c|c|c|c|c|}
\hline G & $\mathrm{R}^{2}$ & $F$ & F crítico & Índice \\
\hline 1 & 0.961 & 199.7 & $\begin{array}{c}4.72 \\
\text { g.I. }(3 ; 24)\end{array}$ & $\begin{array}{c}\mathrm{I}_{1}=0.148[\mathrm{Mg}]+16.47[\mathrm{Zn}]+12.47[\mathrm{Ba}]-1.27 \\
\mathrm{Z}_{1}=0.587 \mathrm{Z}_{\mathrm{Mg}}+0.218 \mathrm{Z}_{\mathrm{Zn}}+0.236 \mathrm{Z}_{\text {ва }}\end{array}$ \\
\hline 2 & 0.961 & 140.9 & $\begin{array}{c}4.26 \\
\text { g.l. }(4 ; 23)\end{array}$ & $\begin{array}{c}\mathrm{I}_{2}=1.97\left[\mathrm{PO}_{4}\right]+0.042[\text { Turb }]+0.28[\mathrm{Na}]+0.17\left[\mathrm{NO}_{3}\right]-1.163 \\
\mathrm{Z}_{2}=0.613 \mathrm{Z}_{\mathrm{PO4}}+0.217 \mathrm{Z}_{\text {Turb }}+0.194 \mathrm{Z}_{\mathrm{Na}}+0.176 \mathrm{Z}_{\mathrm{NO} 3}\end{array}$ \\
\hline 3 & 0.893 & 67.03 & $\begin{array}{c}4.72 \\
\text { g.I. }(3 ; 24)\end{array}$ & $\begin{array}{c}\mathrm{I}_{3}=0.029\left[\mathrm{SO}_{4}\right]+13.99[\mathrm{Mn}]+0.046[\mathrm{Cor}]-1.26 \\
\mathrm{Z}_{3}=0.194 \mathrm{Z}_{\mathrm{SO}}+0.431 \mathrm{Z}_{\mathrm{Mn}}+0.473 \mathrm{Z}_{\mathrm{Cor}}\end{array}$ \\
\hline
\end{tabular}

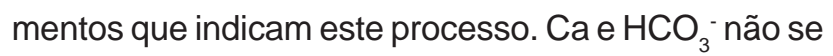
mostraram bons indicadores para as rochas carbonáticas do Grupo Vazante, pois também são influenciados pelas calcitas presentes nos filitos carbonosos da Formação Paracatu. $\mathrm{O} \mathrm{I}_{2}$ caracteriza a intensidade do impacto da entrada dos esgotos domésticos nos sistemas aquáticos, servindo como um IQA Índice de Qualidade de Água para as bacias estudadas, sendo que, os melhores indicadores são os parâmetros $\mathrm{PO}_{4}^{3-}, \mathrm{NO}_{3}^{-}$, turbidez e $\mathrm{Na}$. Entre os compostos nitrogenados, $\mathrm{NO}_{3}$ - mostrou ser um melhor indicador em relação à $\mathrm{NH}_{3}^{3}$ devido as características oxidantes das águas superficiais. $\mathrm{O} \mathrm{I}_{3}$ demonstra a intensidade de mineralização da água pela oxidação da pirita tendo como melhores parâmetros indicadores o $\mathrm{SO}_{4}{ }^{2-}, \mathrm{Mn}$ e cor. Os valores dos índices obtidos para cada ponto amostrado estão descritos na Figura 3.

Os pontos que são mais influenciados pela dissolução das rochas carbonáticas, por apresentarem maiores valores em $\mathrm{I}_{1}$, estão localizados no ribeirão Santa Catarina cuja unidade geológica predominante é o grupo Vazante (Figura 3 a e b). Os pontos que apresentaram anomalias geoquímicas de Zn foram BRSC6 $(48.8 \mu \mathrm{g} / \mathrm{L})$ e BRE10 (47.6 $\mu \mathrm{g} / \mathrm{L})$, estando situados à jusante das mineradoras Vazante e Morro Agudo, respectivamente. BRSC6 encontra-se na Formação Morro do Calcário, composta por dolomitos estromatolíticos, dolarenitos e doloruditos que hospedam as mineralizações de galena e esfalerita e BRE10 encontra-se na Formação Serra do Poço Verde, caracterizada por uma sequência de dolomitos algais onde ocorrem as mineralizações de willemita e calamina. O Ba, diferentemente do $\mathrm{Zn}$, apresentou concentrações anômalas nos pontos BRSC6 $(60.7 \mu \mathrm{g} / \mathrm{L})$ e BRSC7 $(71.5$ $\mu \mathrm{g} / \mathrm{L})$, situados na formação Serra do Poço Verde Grupo Vazante.

Os pontos que são mais influenciados pela oxidação da pirita encontram-se no alto curso do córrego Rico, cuja nascente encontra-se em Morro do Ouro Formação Paracatu, local onde ocorrem mineralizações de sulfetos de ferro e arsênio, apresentando elevados valores no $\mathrm{I}_{3}$ (Figura 3 b). Essas águas apresentaram concentrações elevadas de $\mathrm{SO}_{4}{ }^{2-} \mathrm{em} \mathrm{BCR} 1(21 \mathrm{mg} / \mathrm{L})$, BCR2 (23 mg/L) e BCR4 (17 mg/L), de Mn em BCR2 $(110 \mu \mathrm{g} / \mathrm{L})$ e BCR4 $(123 \mu \mathrm{g} / \mathrm{L})$ e de Fe em BCR1 (430 $\mu \mathrm{g} / \mathrm{L})$ e BCR2 $(420 \mu \mathrm{g} / \mathrm{L})$.

Os pontos que são mais influenciados pela entrada de esgotos domésticos, apresentando elevados valores no $\mathrm{I}_{2}$, estão situados no trecho em que o córrego Rico atravessa a cidade de Paracatu, sendo esta a cidade que apresenta maior número de habitantes e rio que tem a menor densidade de drenagem e,

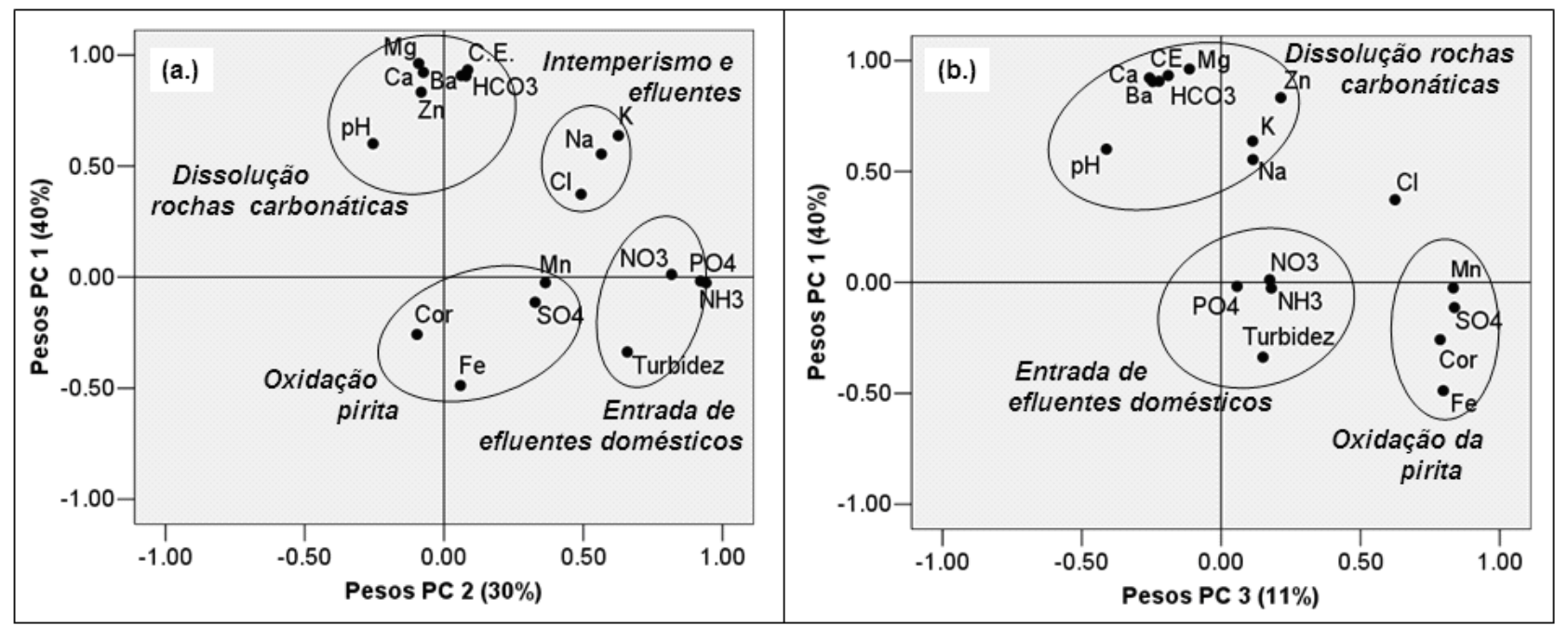

Figura 2 - Agrupamento dos parâmetros de acordo com os pesos obtidos na APC. a.) PC1 x PC2 e b.) PC1 x PC3. 


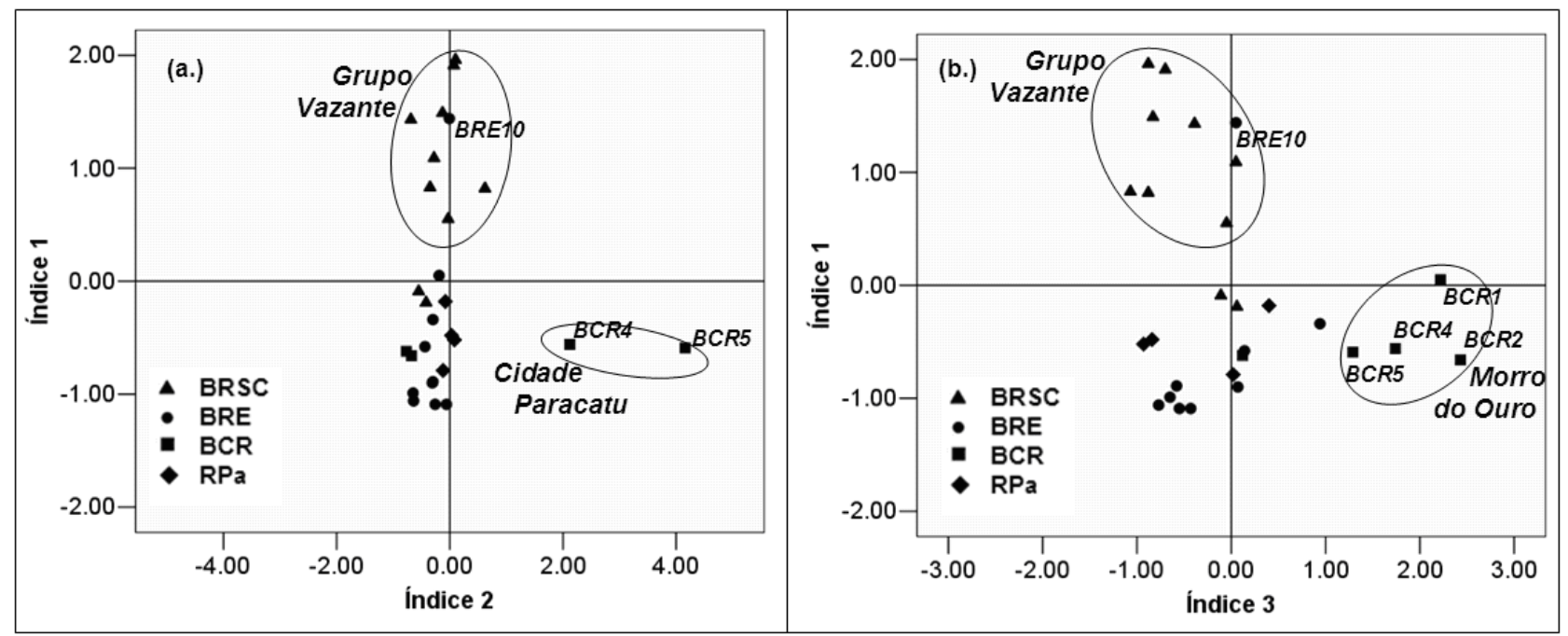

Figura 3 - Agrupamento dos pontos de acordo com os valores obtidos para cada índice calculado. a) Índice 1 x Índice 2 e b) Índice 1 x Índice 3.

consequentemente, a menor vazão e capacidade de diluir os efluentes lançados (Figura $3 \mathrm{a}$ ). $\mathrm{NH}_{3}$ apresentou concentrações anômalas em BCR4 (1.8 mg/L) e BCR5 (2.8 mg/L). $\mathrm{NO}_{3}$ - obteve elevadas concentrações em BCR5 (5.6 mg/L) e $\mathrm{PO}_{4}{ }^{3-}$ em BCR4 (1.0 mg/L) e BCR5 (1.4 mg/L). Os rios situados próximos às demais cidades (Vazante, Lagamar e Guarda-Mor) não apresentaram anomalias destes parâmetros, mostrando que ainda não são influenciados pela ausência de um sistema de coleta e tratamento de esgotos que contemplem toda a cidade.

$\mathrm{Na}$ e K apresentaram anomalias geoquímicas,

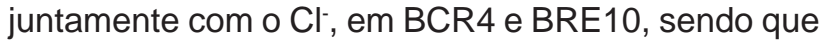
o primeiro ponto está associado à entrada de esgoto doméstico no córrego Rico, proveniente da cidade de Paracatu e o segundo está relacionado à disposição de efluentes da estação de tratamento de esgotos da mineradora Morro Agudo, no ribeirão Traíras.

\section{Classificação da água}

A classificação da água quanto ao processo hidrogeoquímico predominante foi realizada através da análise de agrupamento hierárquico, utilizando os valores dos três índices calculados para cada ponto amostrado. A aplicação deste método permitiu a criação de quatro classes, conforme ilustra a Figura 4.

A classe A é composta por amostras da bacia do rio Escuro (BRE1, BRE2, BRE3, BRE4, BRE5, BRE6, BRE7, BRE9 e BRE13) e do rio Paracatu (RPa1, $\mathrm{RPa} 2, \mathrm{RPa}$ e RPa4), com predominância de águas de baixa mineralização com C.E. $<98 \mu \mathrm{S} / \mathrm{cm}, \mathrm{Ca}+\mathrm{Mg}<$ $11 \mathrm{mg} / \mathrm{L} \mathrm{e} \mathrm{HCO}_{3}^{-}<61 \mathrm{mg} / \mathrm{L}$. Estas amostras estão situadas em regiões de filitos e quartzitos da formação Paracatu, com exceção dos pontos BRSC4 e BRSC9.

As classes $\mathrm{B}$ e $\mathrm{C}$ são compostas por amostras do córrego Rico. A classe B está relacionada com pon- tos BCR1 e BCR2 de água sulfatada, localizadas no alto curso do córrego Rico. Apresentou concentrações de $\mathrm{SO}_{4}{ }^{2-}>21 \mathrm{mg} / \mathrm{L}, \mathrm{Ca}+\mathrm{Mg}<8.2 \mathrm{mg} /{\mathrm{L} \mathrm{e} \mathrm{HCO}_{3}-22}^{-}<2$ $\mathrm{mg} / \mathrm{L}$. A classe $\mathrm{C}$ representa os pontos BCR4 e BCR5 cuja composição geoquímica é influenciada pela entrada de efluentes domésticos não tratados, provenientes da cidade de Paracatu. Apresentam concentrações de $\mathrm{NH}_{3}>1.8 \mathrm{mg} / \mathrm{L} ; \mathrm{NO}_{3}{ }^{-}>0.6 \mathrm{mg} / \mathrm{L} ; \mathrm{PO}_{4}{ }^{3-}>1.0 \mathrm{mg} / \mathrm{L} \mathrm{e}$ turbidez > 15 FTU.

A classe D apresenta amostras de elevada mineralização carbonática, agrupando amostras do ribeirão Santa Catarina (BRSC1, BRSC2, BRSC3, BRSC5, BRSC6, BRSC7, BRSC8 e BRSC10) e um ponto da bacia do rio Escuro (BRE10). Este grupo apresentou C.E. $>156 \mu \mathrm{S} / \mathrm{cm}, \mathrm{Ca}+\mathrm{Mg}>25 \mathrm{mg} / \mathrm{L} \mathrm{e} \mathrm{HCO}_{3}$ $>148 \mathrm{mg} / \mathrm{L}$. Essas amostras estão situadas na unidade geológica do grupo Vazante, composta por rochas carbonáticas.

As distintas unidades geológicas são responsáveis pela formação de águas de composição diferente, sendo que cada bacia apresenta uma característica predominante de acordo com a sua geologia. A bacia do rio Escuro apresenta água de baixa mineralização devido à baixa solubilidade dos quartzitos e filitos da formação Paracatu. A bacia do ribeirão Santa Catarina apresenta águas de elevada mineralização carbonática devido ao processo de dissolução das rochas carbonáticas do grupo Vazante. A bacia do córrego Rico apresenta águas de elevada mineralização sulfatada resultantes do processo de oxidação da pirita, presente no Morro do Ouro. As interferências antrópicas se resumem na entrada de esgotos domésticos nos sistemas aquáticos, provenientes da cidade de Paracatu e de efluentes da ETE da mineradora Morro Agudo. 


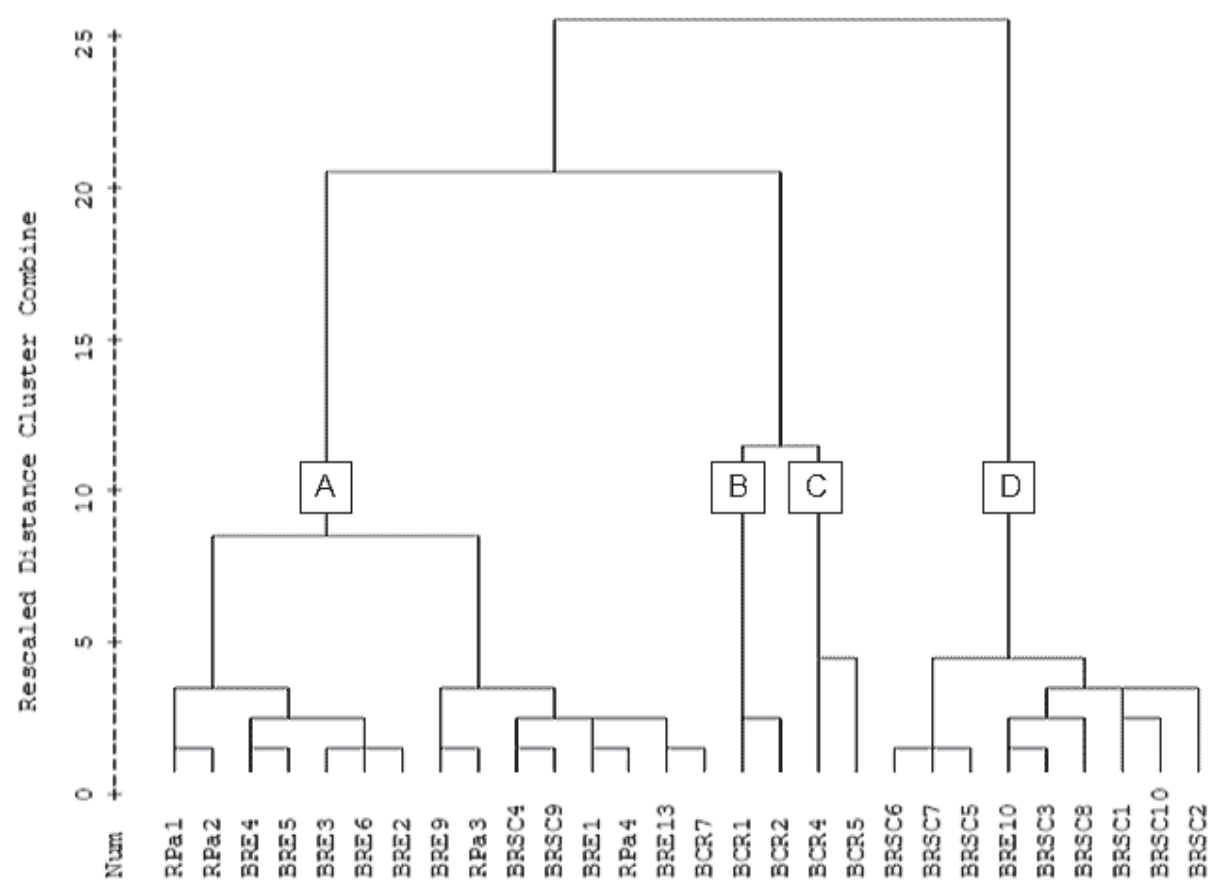

Figura 4 - Dendograma indicando o agrupamento dos pontos amostrados de acordo com os índices calculados. Método de Ward e distância Euclidiana.

\section{CONCLUSÃO}

A utilização da análise multivariada na interpretação dos dados permitiu construir um modelo para a interpretação dos principais processos hidrogeoquímicos das bacias hidrográficas estudadas, contribuindo com a distinção dos parâmetros de origem geológica dos antrópicos e a classificação da água de acordo com sua composição predominante. Possibilitou identificar como as diferentes formações geológicas influenciam na composição química da água (baixa mineralização, elevada mineralização carbonática ou sulfatada), além de determinar a intensidade dos impactos das cidades na qualidade dos sistemas aquáticos.

A metodologia empregada para a construção do modelo geoquímico, proposto neste trabalho, pode servir de base para outras regiões, sendo que a determinação das fontes, dos índices geoquímicos e das classes de água deve ser revista temporalmente, tendo em vista as constantes alterações relacionadas à ocupação e uso do solo e a atualização da base de dados.

\section{AGRADECIMENTOS}

Ao CNPq (Conselho Nacional de Desenvolvimento Científico e Tecnológico) pela concessão de bolsa de estudo para Mestrado e pelo auxilio financeiro, essencial à execução deste projeto (CNPq-Processo 555953-06-08).

\section{REFERÊNCIAS BIBLIOGRÁFICAS}

Andrews-Jones, D.A. 1968. The application of geochemical techniques to mineral exploration. Mineral Industries Bulletin. Colorado School of Mines. 2(6):1-31.

Ball, R.O. \& Church, R.L. 1980. Water quality indexing and scoring. Journal of the Environmental Engineering Divison. 106(4):757-771.

Boaventura, G.R.; Brito, V.E.B. \& Bispo, R.S. 1998. Caracterização Geoquímica de Águas da Bacia do Rio São Bartolomeu-DF. Revista da Escola de Minas. 51(3):57-61.

Brasil. 1988. Departamento Nacional de Produção Mineral. Principais depósitos minerais do Brasil. CPRM, Brasília, 670p.

Brasil. 1996. Ministério do Meio Ambiente. Plano Diretor de Recursos Hídricos da Bacia do Rio Paracatu. Brasília: PLANPAR R2 - Relatório da situação atual. Consórcio MAGNA/DAM/EYSER. 212p

Brasil. 1998. Ministério de Meio Ambiente. Plano diretor de recursos hídricos da Bacia do Rio Paracatu: PLANPAR R7 - Relatório Síntese. Consórcio MAGNA/DAM/EYSER. 135p

Brasil. 2004. Ministério da Saúde. Portaria n 518/04. Estabelece os procedimentos e responsabilidades relativos ao controle e vigilância da qualidade da água para consumo humano e seu padrão de potabilidade, e dá outras providências. 
Bricker, O.P. \& Jones, B.F. 1995. Main factors affecting the composition of natural waters. In: Salbu, B., Steinnes, E (ed). Trace Elements in Natural Waters. CRC Press. Boca Raton. 1-20pp.

Campos Neto, M.C. 1984. Litoestratigrafia, relações estratigráficas e evolução paleogeográfica dos grupos Canastra e Paranoá (região de VazanteLagamar, MG). Revista Brasileira de Geociências. 14(2):81-91.

Carpenter, S.R.; Caraco, N.F.; Correl, D.L.; Howarth, R.W.; Sharpley, A.N. \& Smith, V.H. 1998. Nonpoint pollution of surface waters with phosphorus and nitrogen. Ecological Applications. 8(3):559-568.

CETESB. 1974. Água: qualidade padrões de potabilidade e poluição. CETESB, São Paulo. 208 p.

Chen, K.; Jiao, J.J.; Huang, J. \& Huang, R. 2007. Multivariate statistical evaluation of trace elements in groundwater in a costal area in Shenzhen, China. Environmental Pollution. 147(3):771-780.

CNM. 2007. Informações Municipais. Confederação Nacional de Municípios. Disponível em: <http:// www.cnm.org.br/> Acesso em: $20 \mathrm{dez} 2007$

Davis, J.C. 1986. Statistics and data analysis in geology. $2^{\circ}$ Edição. John Wiley \& Sons. New York. 646 p.

Farnham, I.M.; Johannesson, K.H.; Singh, A.K.; Hodge, V.F. \& Stetzenbach, K.J. 2003. Factor analytical approaches for evaluating groundwater trace element chemistry data. Analytica Chimica Acta. 490:123138.

Freitas-Silva, F.H. \& Dardenne, M.A. 1992. Controles litoestruturais do depósito de ouro do Morro do Ouro, Paracatu, MG. Revista Escola de Minas. Ouro Preto (MG). 45(3):216-216.

Haase, J.; Krieger, J.A. \& Possoli, S. 1989. Estudo da viabilidade do uso da técnica fatorial como um instrumento na interpretação de qualidade das águas da bacia hidrográfica do Guaiba, RS, Brasil. Ciência e Cultura. 41:576-582.

Licht, O.A.B. 1998. Prospecção Geoquímica: Princípios, Técnicas e Métodos. CPRM. Rio de Janeiro. 236p.

Lohani, B.N. \& Mustapha, N. 1982. Indices for water quality assessment in river: a case study of the Linggi river in Malaysia. Water Supply and Management. 6:545-555.

Martinelli, J. A.; Krushce, A. V.; Vicgoria, R. L.; De Camargo, P.B.; Bernardes, M.; Ferraz, E. S.; De Moraes, J. M. \& Ballester, M.V. 2004. Effects of Sewage on the Chemical Composition of Piracicaba River, Brazil. Water, Air, \& Soil Pollution. 110(1):6779

Olías, M.; Nieto, J.M; Sarmiento, A.M; Cerón, J.C \& Cánovas, C.R. 2004. Seasonal water quality variations in a river affected by acid mine drainage: the Odiel River (South West Spain). Science of the Total Environment. 333:267-281

Ott, W.R. 1978. Environmental indices: theory and practice. Michigam. Ann Arbor Science Publ.

Petersen, W.; Bertino, L.; Callies, U. \& Zorita, E. 2001. Process identification by principal component analysis of river water-quality data. Ecological Modelling. 138:193-213.

Pieterse, N.M.; Bleuten, W. \& Jorgensen, S.E. 2003. Contribution of point sources and diffuse sources to nitrogen and phosphorus loads in lowland river tributaries. Journal of Hidrology. 271:213-225.

Rose, A.W.; Hawkes, H.E. \& Webb, J.S. 1979. Geochemistry in mineral exploration. $2^{\circ}$ Edição. Academic Press. New York. 657p.

Shoji, H.; Yamamoto, T. \& Nakamura, T. 1966. Factor analysis on stream pollution of the Yodo River systems. Air and Water Pollution. 10:291-299.

Toledo, LG. \& Nicolella, G. 2002. Índice de qualidade de água em microbacia sob uso agrícola e urbano. Scientia Agricola. 59(1):181-186.

Valeriano, C.M.; Dardenne, M.A.; Fonseca, M.A.; Simões, L.S. \& Seer, H.J. 2004. A evolução tectônica da Faixa Brasília. In: Geologia do Continente Sul Americano: Evolução da Obra de Fernando Flavio Marques de Almeida. Beca. São Paulo. 355-368pp.

Van Andel, T.H.; Zagger, E.E \& Demitrack, A. 1997. Land use and soil erosion in prehistorci and historical Greece. In: Goudie, A. \& Alexander, D.E. (ed.). The human impact reader: Reading and Case Studies. Blackwell Publishing. 94-177pp.

Voudouris, K.; Lambrakis, N.; Papatheodorou, G. \& Daskalaki, P. 1997. An application of factor analysis for the study of the hydrogeological conditions in Plio-Pleistocene aquifers of NW Achaia (NW Peloponnesus, Greece). Math. Geology. 29(1):4359.

Ward, J.H. 1963. Hierarchical grouping to optimize an objective function. Journal of American Statistics Association. 69:236-244.
Submetido: Novembro/2008 Revisado: Novembro/2009 Aceito: Agosto/2010 